\title{
Namenregister zu Band 95
}

A

Albrecht 129 Allmaras 276 Amsler 338 Apetz 168 Arnold 361 Auffinger 241, 366

B

Bair 174

Barkan 237

Bartels 177, 299, 301, 360

Böck 1

Braun 342, 348

Bruckmann 109

Bruckner 206

Brüning 351

Bucklers 338, 345, 352

C

Cibis 344

Comberg 338, 350, 351

Custodis 259

D

Davids 341 Döhmen 357 Droescher 351

E Engelking 351, 352, 356,

359 Erggelet 355 Eversheim 363

F Fischer, Fr. 346 Fleischer 163, 166, 170, 171, 356 Friemann 297, 298 Fuchs 336

G

Gasteiger 167, 170 Gerlach 339 Gifford Ill Gilbert 341, 342 Graff 350 Groenouw 110 Grüter 336, 337

$\mathrm{H}$

Harms 349 Hartlinger 356 Heinsius 349 Heinz 365 Hertel 368 Herzau 353 Hesseberg 139 vom Hofe 339 Hoffmann-Rötzel 323 Hollwich 164

I

Imre 340

$\mathrm{J}$

Jaenisch 297, 302, 358 Jäger 164 Jeß 342, 352

$\mathrm{K}$

Kapfhammer 345

Klar 337, 343, 351

Klemens 354

Koch 360

Königer 165

Kreibig 113, 269, 341, 354 
Krückmann 358, 359

Kurz 315

Kyrieleis 343

$\mathrm{L}$

Lauber 342, 352 Lindner 346 Lisch 168 Loepp 188

Löhlein 339, 344, 346, 350

$\mathrm{M}$

Marchesani 341, 359,

360, 361 Matthaei 170 Meesmann 338, 346 Meisner (München) 165 Meissner (Wien) 364

Motolese 338 Müller, H. K. 345, 351 Munzert 169 Mylius 40, 343

N zur Nedden 360 Nolte 298

0 Ohm 200, 297, 299, 303

$\mathrm{P}$

Pantasatos 305 Passow 162 Pautrier 109, 288 Peters 162 (P.) Pick 333

Pillat 64, 340, 343, 347 Plitt 170 Poos 361

$\mathrm{R}$

Raaf 174

Rauh 345

Reichling 346, 354, 355

Reiser 338

Riechert 344

Rieger 347

Rintelen 280, 336

Roggenkämper 297, 301,

302 Rohrschneider 340 Rößler 167

S Saßler 355 Scheerer 343 Scheyhing 168, 357 Schlagenhauff 364 Schlösser 363 Schmelzer 165, 169, 347 Schmidt 343, 344, 350,

356, 358 Schreck 348, 349, 352 Schwickerath 302 Seefelder 346, 348 Seidel 341, 343, 353 Serr

337, 348, 355 Sohr 351 Stahl 176 Steinseifer 51 Stock 166 Süllmann 58

$\mathrm{T}$

Thiel 339, 348 Tchermak-Seysenegg 352

U Urbanek 129, 345

$\mathrm{V}$

Velhagen 344, 359, 360 Vogt 173 Völkel 340

$\mathrm{W}$

Weekers 58 Wegner 349 Werner 108 Wilde 298

Z. Z

Zobel 137 